\title{
Fixed point sets of set-valued mappings
}

\author{
Parunyou Chanthorn ${ }^{1}$ and Phichet Chaoha ${ }^{1,2^{*}}$
}

\author{
${ }^{*}$ Correspondence: \\ phichet.c@chula.ac.th \\ ${ }^{1}$ Department of Mathematics and \\ Computer Science, Faculty of \\ Science, Chulalongkorn University, \\ Bangkok, 10330, Thailand \\ ${ }^{2}$ Centre of Excellence in \\ Mathematics, CHE, Si Ayutthaya Rd. \\ Bangkok, 10400, Thailand
}

\begin{abstract}
We present new results regarding fixed point sets of various set-valued mappings using the concept of fixed point iteration schemes and the newly defined concept of fixed point resolutions. In particular, we prove that the fixed point sets of certain nonexpansive set-valued mappings are contractible.
\end{abstract}

MSC: $47 \mathrm{H} 04 ; 47 \mathrm{HO}$

Keywords: fixed point set; set-valued mapping

\section{Introduction}

In 1973, Bruck [1] gave an intriguing result on the structure of fixed point sets by proving that the fixed point set of a certain nonexpansive mapping is always a nonexpansive retract of its domain. It took almost thirty years before the result was extended to asymptotically nonexpansive mappings in [2]. From the point of view of topological theory, such retraction results enable us to pass various topological properties (for example, connectedness and contractibility) from the domain of the mapping onto its fixed point set. Recently in [3], Chaoha introduced the notion of virtually nonexpansive mappings (which includes various nonexpansive-type mappings) on metric spaces and proved that the fixed point set of a virtually nonexpansive mapping is always a retract of a certain subset called the convergence set. Consequently, Chaoha and Atiponrat [4] extended the notion of virtually nonexpansive mappings on metric spaces to virtually stable mappings on Hausdorff spaces and presented a retraction result similar to [3] for regular spaces. Recently, Chaoha and Chanthorn [5] presented the concept of fixed point iteration schemes that unifies well-known iteration processes (for example, Picard, Mann and Ishikawa iteration processes [6]) and showed that in regular spaces the fixed point set of a certain virtually stable scheme is a retract of its convergence set. Combined with numerous convergence results of iteration processes in the literature, the authors were able to derived some contractibility criteria for fixed point sets of mappings in various situations. For set-valued mappings, fewer results on the structure of fixed point sets have been explored. It was recently proved in [7] that the fixed point set of a certain quasi-nonexpansive set-valued mapping on a $\mathrm{CAT}(0)$ space is always convex and hence contractible. In this work, we use the concept of virtually stable schemes to acquire retraction results for the fixed point sets of set-valued mappings in appropriate settings. Especially, combined with Nadler's result in [8], we obtain a new contractibility criterion for the fixed point set of a certain set-valued $\alpha$-contraction. Then we will construct a sequence of mappings that is not naturally a scheme, but surprisingly yields similar retraction and contractibility results for the

(c) 2015 Chanthorn and Chaoha; licensee Springer. This article is distributed under the terms of the Creative Commons Attribution 4.0 International License (http://creativecommons.org/licenses/by/4.0/), which permits unrestricted use, distribution, and reproduction in any medium, provided you give appropriate credit to the original author(s) and the source, provide a link to the Creative Commons license, and indicate if changes were made. 
fixed point set of a certain nonexpansive set-valued mapping. This immediately calls for the introduction of fixed point resolutions generalizing fixed point iteration schemes at the end of this work.

The paper is organized as follows. In Section 2, we recall the backgrounds used throughout this work. In particular, we present some useful conditions involving set-valued mappings as well as the new concept of $\alpha$-contractive schemes. In Section 3, we first construct, with the aid of Michael's selection theorem, virtually stable schemes for certain set-valued mappings to obtain retraction results for fixed point sets. Then we construct an $\alpha$-contractive scheme for a set-valued $\alpha$-contraction to obtain a new contractibility criterion for its fixed point set. Finally, we construct a sequence of mappings that is not a fixed point iteration scheme, but still induces retraction and contractibility results for the fixed point set of a certain nonexpansive set-valued mapping.

\section{Preliminaries}

For a nonempty set $X$, we let $\mathcal{P}(X)$ be the power set of $X$ and $2^{X}=\mathcal{P}(X)-\{\emptyset\}$. Also, we denote the fixed point sets of a single-valued mapping $f: X \rightarrow X$ and a set-valued mapping $F: X \rightarrow 2^{X}$ by $\operatorname{Fix}(f)=\{x \in X: x=f(x)\}$ and $\operatorname{Fix}(F)=\{x \in X: x \in F(x)\}$, respectively. Throughout this paper, we always assume that every mapping has nonempty fixed point sets.

For a metric space $(X, d), a \in X$, and $A, B \in 2^{X}$, let

- $B(a ; \varepsilon)=\{y \in X: d(a, y)<\varepsilon\}$,

- $D(a ; \varepsilon)=\{y \in X: d(a, y) \leq \varepsilon\}$,

- $S(a ; \varepsilon)=\{y \in X: d(a, y)=\varepsilon\}$,

- $\eta(A ; \varepsilon)=\bigcup_{a \in A} B(a ; \varepsilon)$,

- $d(A, B)=\inf \{d(a, b): a \in A$ and $b \in B\}$,

- $d(a, B)=d(\{a\}, B)=\inf _{b \in B} d(a, b)$,

- $h(A, B)=\sup _{a \in A} d(a, B)$, and

- $\mathrm{CB}(X)=\left\{A \in 2^{X}: A\right.$ is closed and bounded $\}$.

The Hausdorff metric [9] is the mapping $H: \mathrm{CB}(X) \times \mathrm{CB}(X) \rightarrow \mathbb{R}$ defined by

$$
H(A, B)=\inf \{\varepsilon: A \subseteq \eta(B ; \varepsilon) \text { and } B \subseteq \eta(A ; \varepsilon)\}
$$

for each $A, B \in \mathrm{CB}(X)$.

The following facts can be found in $[8,10,11]$.

Lemma 2.1 Let $x, y \in X$ and $A, B \subseteq X$. Then we obtain the following:

(1) $d(x, A) \leq d(x, y)+d(y, A)$.

(2) $d(y, A) \leq \sup _{b \in B} d(b, A)=h(B, A)$ for each $y \in B$.

(3) $H(A, B)=\max \{h(A, B), h(B, A)\}$ for each $A, B \in \mathrm{CB}(X)$.

(4) For each $a \in A$ and $\varepsilon>0$, there is $b \in B$ such that $d(a, b) \leq H(A, B)+\varepsilon$.

For a set-valued mapping $F: X \rightarrow 2^{X}$, let $P_{F}: X \rightarrow \mathcal{P}(X)$ be defined by

$$
P_{F}(x)=\{y \in F(x): d(x, y)=d(x, F(x))\}
$$

for each $x \in X$, and we say that $F$ satisfies 
- the end-point condition [12] if $F(p)=\{p\}$ for each $p \in \operatorname{Fix}(F)$,

- the proximal condition if $P_{F}(x) \neq \emptyset$ for each $x \in X$,

- the Chebyshev condition if $P_{F}(x)$ is a singleton for each $x \in X$.

Notice that every single-valued mapping (considered as a set-valued mapping in the trivial way) satisfies all above conditions.

Remark 2.2 The definitions of the proximal condition and the Chebyshev condition are motivated by the proximal set and the Chebyshev set, respectively, in [13]. When a mapping $F: X \rightarrow 2^{X}$ satisfies the Chebyshev condition, we will identify $P_{F}(x)$ with its element, i.e., $P_{F}$ can be considered as a mapping on $X$ in this case. Note that every set-valued mapping with compact values always satisfies the proximal condition.

Recall that $f: X \rightarrow Y$ is called a selection of the set-valued mapping $F: X \rightarrow 2^{Y}$ if $f(x) \in$ $F(x)$ for each $x \in X$.

Proposition 2.3 IfF $: X \rightarrow 2^{X}$ satisfies the end-point condition and $f: X \rightarrow X$ is a selection of $F$, then $\operatorname{Fix}(F)=\operatorname{Fix}(f)$.

Lemma 2.4 For a metric space $(X, d)$, if $F: X \rightarrow 2^{X}$ satisfies the proximal condition, then we have the following:

(1) $\operatorname{Fix}(F)=\operatorname{Fix}\left(P_{F}\right)$.

(2) $P_{F}$ satisfies the end-point condition.

(3) $P_{F}(x)=F(x) \cap S(x ; d(x, F(x)))$ for each $x \in X$.

(4) If $F$ has closed values, then $P_{F}(x)$ is closed and bounded for each $x \in X$.

Proof (1) and (2) are obvious. (3) is straightforward from the definition of $P_{F}$. (4) follows directly from (3) and the fact that $F(x)$ is closed for each $x \in X$.

We now recall some definitions of continuity for set-valued mappings (see [10] for more details). For our purpose, let $X$ and $Y$ be metric spaces (with no ambiguity, their metrics will be denoted by the same symbol ' $d$ '). A set-valued mapping $F: X \rightarrow 2^{Y}$ is said to be

- upper semi-continuous at $x$ if $\left(x_{n}\right)$ is a sequence in $X$ converging to $x$ and $U$ is an open subset of $Y$ such that $F(x) \subseteq U$, then there exists $N \in \mathbb{N}$ such that $F\left(x_{n}\right) \subseteq U$ for each $n \geq N$,

- lower semi-continuous at $x$ if $\left(x_{n}\right)$ is a sequence in $X$ converging to $x$ and $y \in F(x)$, then there exists a sequence $\left(y_{n}\right)$ in $Y$ such that $y_{n} \in F\left(x_{n}\right)$ and $\left(y_{n}\right)$ converges to $y$,

- H-upper semi-continuous at $x$ if for each $\varepsilon>0$, there is $\delta>0$ such that $h(F(y), F(x))<\varepsilon$ for each $y \in B(x, \delta)$,

- $H$-lower semi-continuous at $x$ if for each $\varepsilon>0$, there is $\delta>0$ such that $h(F(x), F(y))<\varepsilon$ for each $y \in B(x, \delta)$,

- $(H-)$ continuous at $x$ if $F$ is $(H-)$ upper and $(H-)$ lower semi-continuous at $x$.

We say that the set-valued mapping $F: X \rightarrow 2^{Y}$ is (upper, $H$-upper, lower, $H$-lower semi-, $H$-) continuous if it is (upper, $H$-upper, lower, $H$-lower semi-,$H$-) continuous at each point in $X$. Moreover, for $\alpha \in[0,1)$, we say that the mapping $F: X \rightarrow \mathrm{CB}(X)$ is

- an $\alpha$-contraction if $H(F(x), F(y)) \leq \alpha d(x, y)$ for each $x, y \in X$,

- nonexpansive if $H(F(x), F(y)) \leq d(x, y)$ for each $x, y \in X$,

- quasi-nonexpansive if $H(F(x), F(p)) \leq d(x, p)$ for each $x \in X$ and $p \in \operatorname{Fix}(F)$, 
- *-nonexpansive [14] if for each $x, y \in X$ and $u_{x} \in P_{F}(x)$, there is $u_{y} \in P_{F}(y)$ such that $d\left(u_{x}, u_{y}\right) \leq d(x, y)$.

It is not difficult to see that every set-valued $\alpha$-contraction is nonexpansive and $H$ continuous, while every nonexpansive set-valued mapping is quasi-nonexpansive and continuous. Moreover, when $F$ is single-valued, the above definitions of $\alpha$-contraction, nonexpansive mapping and quasi-nonexpansive set-valued mapping coincide with the usual definitions for single-valued mapping.

Lemma 2.5 ([10], Proposition 5.3.42 and Proposition 5.3.43) Let $X$ and $Y$ be metric spaces and $F: X \rightarrow 2^{Y}$ be a mapping.

(1) If $F$ is H-upper semi-continuous and has compact values, then it is upper semi-continuous.

(2) If $F$ is $H$-lower semi-continuous, then it is lower semi-continuous.

Lemma 2.6 ([15], Proposition 2.3) Let $X$ and $Y$ be metric spaces and $F: X \rightarrow 2^{Y}$ be lower semi-continuous. If a mapping $G: X \rightarrow 2^{Y}$ satisfies $\overline{G(x)}=\overline{F(x)}$ for each $x \in X$, then $G$ is lower semi-continuous.

Lemma 2.7 ([10], Proposition 5.3.20) Let $X$ be a metric space and $Y$ be a Banach space. Assume that $F: X \rightarrow 2^{Y}$ and $G: X \rightarrow 2^{Y}$ are lower semi-continuous. If $G$ has open convex values and $F(x) \cap G(x) \neq \emptyset$ for each $x \in X$, then the mapping $\Phi: X \rightarrow 2^{Y}$ defined by

$$
\Phi(x)=F(x) \cap G(x)
$$

for each $x \in X$ is lower semi-continuous.

Lemma 2.8 Every selection of a quasi-nonexpansive set-valued mapping on a metric space satisfying the end-point condition is quasi-nonexpansive.

Proof It is straightforward from the definition.

Lemma 2.9 Let $X$ be a metric space. If $F: X \rightarrow 2^{X}$ is $*$-nonexpansive with closed values and satisfies the proximal condition, then the mapping $P_{F}: X \rightarrow \mathrm{CB}(X)$ is nonexpansive.

Proof Let $x, y \in X$ and $\varepsilon>0$. For each $z \in P_{F}(x)$, there is $u_{y} \in P_{F}(y)$ such that

$$
z \in B\left(u_{y} ; d(x, y)+\varepsilon\right) \subseteq \bigcup_{u \in P_{F}(y)} B(u ; d(x, y)+\varepsilon)=\eta\left(P_{F}(y) ; d(x, y)+\varepsilon\right)
$$

Thus, $P_{F}(x) \subseteq \eta\left(P_{F}(y) ; d(x, y)+\varepsilon\right)$. Similarly, we have $P_{F}(y) \subseteq \eta\left(P_{F}(x) ; d(x, y)+\varepsilon\right)$. Therefore, since $\varepsilon$ is arbitrary, $H\left(P_{F}(x), P_{F}(y)\right) \leq d(x, y)$.

We will see later that the continuity of the mapping $P_{F}$ plays a crucial role in proving the main result when the set-valued mapping $F$ satisfies the Chebyshev condition. Also in Theorem 2.1.1 [16], it is shown, by using the compactness of the unit disc of the Euclidean space, that every continuous mapping $F$ from the Euclidean space $\mathbb{R}^{n}$ into $2^{\mathbb{R}^{n}}$ satisfying the Chebyshev condition induces the continuity of $P_{F}$. Therefore, to generalize such a result, we will consider a continuous set-valued mapping on a compact metric space as follows. 
Lemma 2.10 Let $X$ be a compact metric space, $p \in X$ and $\left(x_{n}\right)$ be a sequence in $X$. If every convergent subsequence of $\left(x_{n}\right)$ converges to $p$, then so does $\left(x_{n}\right)$.

Proof Suppose that $\left(x_{n}\right)$ does not converge to $p$. Then there exist a neighborhood $U$ of $p$ and a subsequence $\left(x_{n_{k}}\right)$ of $\left(x_{n}\right)$ such that $x_{n_{k}} \notin U$ for each $k \in \mathbb{N}$. By the compactness of $X$, the sequence $\left(x_{n_{k}}\right)$ has a convergent subsequence which is also a subsequence of $\left(x_{n}\right)$, say $\left(x_{m_{k}}\right)$. Hence, $\left(x_{m_{k}}\right)$ converges to a point $p$ in $X$ by the assumption, and we get a contradiction.

Theorem 2.11 Let $X$ be a compact metric space. If $F: X \rightarrow 2^{X}$ is continuous, satisfies the Chebyshev condition and has closed values, then $P_{F}: X \rightarrow X$ is continuous.

Proof Let $\left(x_{n}\right)$ be a sequence in $X$ converging to $x_{0} \in X$. Suppose that $\left(P_{F}\left(x_{n_{k}}\right)\right)$ is a subsequence of $\left(P_{F}\left(x_{n}\right)\right)$ converging to a point $p \in X$. By the upper semi-continuity of $F$, for each $\varepsilon>0$, there is $N \in \mathbb{N}$ such that

$$
P_{F}\left(x_{n_{k}}\right) \in F\left(x_{n_{k}}\right) \subseteq \eta\left(F\left(x_{0}\right) ; \varepsilon\right)
$$

for each $k \geq N$. Hence, $\lim _{k \rightarrow \infty} d\left(P_{F}\left(x_{n_{k}}\right), F\left(x_{0}\right)\right)=0$ and

$$
d\left(p, F\left(x_{0}\right)\right) \leq \limsup _{k \rightarrow \infty}\left[d\left(p, P_{F}\left(x_{n_{k}}\right)\right)+d\left(P_{F}\left(x_{n_{k}}\right), F\left(x_{0}\right)\right)\right]=0 .
$$

Thus, $p \in F\left(x_{0}\right)$ since $F\left(x_{0}\right)$ is closed. Also, for each $a \in F\left(x_{n_{k}}\right)$ and $k \in \mathbb{N}$,

$$
\begin{aligned}
d\left(p, x_{0}\right) & \leq d\left(p, P_{F}\left(x_{n_{k}}\right)\right)+d\left(P_{F}\left(x_{n_{k}}\right), x_{0}\right) \\
& \leq d\left(p, P_{F}\left(x_{n_{k}}\right)\right)+d\left(x_{0}, x_{n_{k}}\right)+d\left(x_{n_{k}}, P_{F}\left(x_{n_{k}}\right)\right) \\
& \leq d\left(p, P_{F}\left(x_{n_{k}}\right)\right)+d\left(x_{0}, x_{n_{k}}\right)+d\left(x_{n_{k}}, a\right) \\
& \leq d\left(p, P_{F}\left(x_{n_{k}}\right)\right)+2 d\left(x_{0}, x_{n_{k}}\right)+d\left(x_{0}, P_{F}\left(x_{0}\right)\right)+d\left(P_{F}\left(x_{0}\right), a\right) .
\end{aligned}
$$

Since $F$ is lower semi-continuous and $P_{F}\left(x_{0}\right) \in F\left(x_{0}\right)$, there is a sequence $\left(y_{n_{k}}\right)$ in $X$ such that $y_{n_{k}} \in F\left(x_{n_{k}}\right)$ and $\left(y_{n_{k}}\right)$ converges to $P_{F}\left(x_{0}\right)$. Consequently,

$$
\begin{aligned}
d\left(p, x_{0}\right) & \leq \limsup _{k \rightarrow \infty}\left[d\left(p, P_{F}\left(x_{n_{k}}\right)\right)+2 d\left(x_{0}, x_{n_{k}}\right)+d\left(x_{0}, P_{F}\left(x_{0}\right)\right)+d\left(P_{F}\left(x_{0}\right), y_{n_{k}}\right)\right] \\
& =d\left(x_{0}, P_{F}\left(x_{0}\right)\right) \\
& =d\left(x_{0}, F\left(x_{0}\right)\right)=\inf _{y \in F\left(x_{0}\right)} d\left(x_{0}, y\right) \leq d\left(x_{0}, p\right) .
\end{aligned}
$$

It follows that $p \in F\left(x_{0}\right) \cap S\left(x_{0} ; d\left(x_{0}, F\left(x_{0}\right)\right)\right)=P_{F}\left(x_{0}\right)$ by Lemma 2.4(3), and hence $p=$ $P_{F}\left(x_{0}\right)$ by the Chebyshev condition on $F$. This proves that each convergent subsequence of $\left(P_{F}\left(x_{n}\right)\right)$ converges to the same point $P_{F}\left(x_{0}\right)$. Then, by Lemma 2.10 , the sequence $\left(P_{F}\left(x_{n}\right)\right)$ converges to $P_{F}\left(x_{0}\right)$. Therefore, the mapping $P_{F}$ is continuous.

Recall that a metric space $X$ is said to be metrically convex [17] if for each $x, y \in X$ with $x \neq y$, there exists an element $z \in X$ such that $x \neq z, y \neq z$, and $d(x, y)=d(x, z)+d(z, y)$. Notice that every linear space is metrically convex. 
Lemma 2.12 ([17]) Let $X$ be a complete and metrically convex metric space. If $A$ is a nonempty closed subset of $X$, then for each $x \in A$ and $y \notin A$, there exists $z \in \partial A$ (the boundary of $A$ ) such that

$$
d(x, z)+d(z, y)=d(x, y) .
$$

Lemma 2.13 Let $X$ be a complete and metrically convex metric space, $x, y \in X$ and $s, t \in$ $[0, \infty)$. Then we have $H(D(x ; s), D(x ; t))=|s-t|$. Moreover, if $X$ is a normed space (over $\mathbb{R})$, then $H(D(x ; t), D(y ; t))=\|x-y\|$.

Proof WLOG, we assume that $s \leq t$. Since $D(x ; s) \subseteq D(x ; t)$, we have

$$
h(D(x ; s), D(x ; t))=\sup _{a \in D(x ; s)} d(a, D(x ; t))=0,
$$

and hence $H(D(x ; t), D(x ; s))=h(D(x ; t), D(x ; s))$ by Lemma 2.1(3). It is easy to see that $t-s \leq$ $d(y, z)$ for each $y \in S(x ; t)$ and $z \in D(x ; s)$. Also,

$$
t-s \leq \inf _{z \in D(x ; s)} d(y, z)=h(y, D(x ; s)) \leq h(D(x ; t), D(x ; s)) .
$$

On the other hand, for each $y \in S(x ; t)$, there exists $z \in S(x ; s)$ such that $d(x, z)+d(z, y)=$ $d(x, y)$ by Lemma 2.12 . Consequently,

$$
h(y, D(x ; s)) \leq d(y, z) \leq t-s
$$

for each $y \in S(x ; t)$. Then $h(D(x ; t), D(x ; s)) \leq t-s$.

Now if $X$ is a normed space and $x \neq y$, let $a=x+\frac{t}{\|x-y\|}(x-y) \in S(x ; t)$. Then

$$
\begin{aligned}
\|x-y\|+t & =\left(1+\frac{t}{\|x-y\|}\right)\|x-y\| \\
& =\left\|(x-y)+\frac{t}{\|x-y\|}(x-y)\right\| \\
& =\|a-y\| \\
& \leq\|a-z\|+\|z-y\| \leq\|a-z\|+t
\end{aligned}
$$

for each $z \in D(y ; t)$. Therefore, $\|x-y\| \leq\|a-z\|$ for each $z \in D(y ; t)$, and hence

$$
\|x-y\| \leq \inf _{z \in D(y ; t)}\|a-z\|=d(a, D(y ; t)) \leq h(D(x ; t), D(y ; t)) \leq H(D(x ; t), D(y ; t)) .
$$

On the other hand, let $a \in D(x ; t)$ and $\varepsilon>0$. Consider the point $b=a+y-x$. Then $\|a-b\|=$ $\|x-y\|$ and $\|b-y\|=\|a-x\| \leq t$. Hence, we have

$$
a \in B(b ;\|x-y\|+\varepsilon), \quad b \in D(y ; t), \quad \text { and } \quad a \in \eta(D(y ; t) ;\|x-y\|+\varepsilon) .
$$

Consequently, $D(x ; t) \subseteq \eta(D(y ; t) ;\|x-y\|+\varepsilon)$. By a similar argument, we can show that $D(y ; t) \subseteq \eta(D(x ; t) ;\|x-y\|+\varepsilon)$. It follows that $H(D(x ; t), D(y ; t)) \leq\|x-y\|+\varepsilon$, and since $\varepsilon$ is arbitrary, $H(D(x ; t), D(y ; t)) \leq\|x-y\|$. 
The assumption that $X$ is a normed space in the previous lemma is necessary as shown in the following example.

Example 2.14 Consider $[0,1]$ with the standard metric. Let $x=0, y=1$, and $t=1$. Then $H(D(x ; t), D(y ; t))=0$ but $|x-y|=1$.

The following theorem follows directly from the classical Michael's selection theorem ([15], Theorem 3.2").

Theorem 2.15 Every lower semi-continuous set-valued mapping from a metric space into a Banach space with closed and convex values admits a continuous selection.

In a (real) Banach space $(X,\|\cdot\|)$, we let

$$
\mathrm{CC}(X)=\left\{A \in 2^{X}: A \text { is convex }\right\} \quad \text { and } \quad \mathrm{CCB}(X)=\mathrm{CB}(X) \cap \mathrm{CC}(X)
$$

where $\mathrm{CCB}(X)$ is considered as a subspace of the metric space $(\mathrm{CB}(X), H)$.

Lemma 2.16 Let $X$ be a closed subset of a Banach space $E$. Assume that $f: E \rightarrow E$ and $\varphi: E \rightarrow(0, \infty)$ are continuous mappings.

(1) The mapping $\bar{\Phi}: E \rightarrow 2^{E}$ defined by $\bar{\Phi}(x)=D(f(x) ; \varphi(x))$ for each $x \in E$ is lower semi-continuous.

(2) The mapping $\Phi: E \rightarrow 2^{E}$ defined by $\Phi(x)=B(f(x)$; $\varphi(x))$ for each $x \in E$ is lower semi-continuous.

(3) If $F: X \rightarrow \mathrm{CC}(X)$ is a lower semi-continuous mapping satisfying $\Phi(x) \cap F(x) \neq \emptyset$ for each $x \in X$, then there is a continuous mapping $g: X \rightarrow X$ such that $g(x) \in \bar{\Phi}(x) \cap F(x)$ for each $x \in X$.

Proof (1) Let $x \in E$ and $\varepsilon>0$. Since $f$ and $\varphi$ are continuous, there is $\delta>0$ such that

$$
\|f(x)-f(y)\|<\frac{\varepsilon}{2} \quad \text { and } \quad|\varphi(x)-\varphi(y)|<\frac{\varepsilon}{2}
$$

for each $y \in B(x ; \delta)$. Thus, by Lemma 2.13,

$$
\begin{aligned}
H(\bar{\Phi}(x), \bar{\Phi}(y)) \leq & H(D(f(x) ; \varphi(x)), D(f(x) ; \varphi(y))) \\
& +H(D(f(x) ; \varphi(y)), D(f(y) ; \varphi(y))) \\
= & |\varphi(x)-\varphi(y)|+\|f(x)-f(y)\|<\varepsilon
\end{aligned}
$$

for each $y \in B(x ; \delta)$. Therefore, $\bar{\Phi}$ is $H$-continuous and hence lower semi-continuous by Lemma 2.5(2).

(2) It follows directly from (1) and Lemma 2.6.

(3) By (2), the restriction $\left.\Phi\right|_{X}: X \rightarrow 2^{E}$ is lower semi-continuous. For each $x \in X$, since $\Phi(x)$ and $F(x)$ are convex, so is $\overline{\Phi(x) \cap F(x)}$. Following Lemma 2.6 and Lemma 2.7, the mapping $\Psi: X \rightarrow \mathrm{CC}(X) \subseteq \mathrm{CC}(E)$ defined by

$$
\Psi(x)=\overline{\Phi(x) \cap F(x)}
$$


for each $x \in X$ is lower semi-continuous. By Theorem 2.15, there is a continuous mapping $g: X \rightarrow E$ such that

$$
g(x) \in \Psi(x) \subseteq \bar{\Phi}(x) \cap \overline{F(x)}=\bar{\Phi}(x) \cap F(x) \subseteq X
$$

for each $x \in X$.

We now recall the notion of fixed point iteration schemes and virtually stable schemes defined in [5]. For a given (nonempty) Hausdorff space $X$ and a sequence $\mathcal{S}=\left(s_{n}\right)$ of mappings on $X$, let $\operatorname{Fix}(\mathcal{S})=\bigcap_{n=1}^{\infty} \operatorname{Fix}\left(s_{n}\right)$ and $\mathrm{C}(\mathcal{S})=\left\{x: \lim _{n \rightarrow \infty} s_{n}(x)\right.$ exists $\}$ denote the fixed point set and the convergence set of $\mathcal{S}$, respectively. We always have $\operatorname{Fix}(\mathcal{S}) \subseteq \mathrm{C}(\mathcal{S})$, and there is a natural mapping $r: C(\mathcal{S}) \rightarrow X$ defined by

$$
r(x)=\lim _{n \rightarrow \infty} s_{n}(x)
$$

for each $x \in X$. Notice that $r$ may not be continuous in general, and $\operatorname{Fix}(\mathcal{S}) \subseteq r(\mathrm{C}(\mathcal{S}))$. For a sequence $\left(f_{n}\right)$ of mappings on $X$, we write $\prod_{i=j}^{n} f_{i}$ for the composition $f_{n} \circ f_{n-1} \circ \cdots \circ f_{j}$. Notice that if $\mathcal{F}=\left(f_{n}\right)$ is a sequence of mappings and $\mathcal{S}=\left(\prod_{i=1}^{n} f_{i}\right)$, then $\operatorname{Fix}(\mathcal{S})=\operatorname{Fix}(\mathcal{F})$. The sequence $\mathcal{S}=\left(\prod_{i=1}^{n} f_{i}\right)$ is said to be a fixed point iteration scheme, or a scheme in short, if $\operatorname{Fix}(\mathcal{S})=r(\mathrm{C}(\mathcal{S}))$.

Definition 2.17 A scheme $\mathcal{S}=\left(\prod_{i=1}^{n} f_{i}\right)$ on $X$ is said to be a virtually stable scheme if for each common fixed point $p$ of $\mathcal{S}$ and each neighborhood $U$ of $p$, there exist a neighborhood $V$ of $p$ and a subsequence $\left(n_{k}\right)$ of $(n)$ such that $\prod_{i=j}^{n_{k}} f_{i}(V) \subseteq U$ for each $k \in \mathbb{N}$ and $j \leq n_{k}$.

Example 2.18 From [5] it follows that the Picard iteration scheme $\left(f^{n}\right)$ for a continuous quasi-nonexpansive mapping $f$ on a metric space is virtually stable.

Recall that a subset $A$ of the space $X$ is said to be a retract of $X$ if there is a continuous $r: X \rightarrow A$ such that $r(a)=a$ for each $a \in A$. Moreover, $r$ is called a retraction. In addition, a (topological) space $X$ is said to be contractible if there are a point $x_{0} \in X$ and a continuous mapping $f: X \times[0,1] \rightarrow X$ such that $f(x, 1)=x$ and $f(x, 0)=x_{0}$ for all $x \in X$. Note that a convex subset of a Banach space and a retract of a contractible space are always contractible.

Theorem 2.19 ([5], Theorem 2.5) Let $X$ be a regular space. If $\mathcal{S}$ is a virtually stable scheme having a continuous subsequence, i.e., there is a subsequence $\left(s_{n_{k}}\right)$ of $\mathcal{S}$ such that each $s_{n_{k}}$ is continuous, then $r: \mathrm{C}(\mathcal{S}) \rightarrow \operatorname{Fix}(\mathcal{S})$ is a retraction.

Next we will introduce the concept of an $\alpha$-contractive scheme on a metric space $X$ which resembles an $\alpha$-contraction in the sense that it is always virtually stable, and when $X$ is complete, its convergence set is the whole space $X$.

Definition 2.20 For $\alpha \in[0,1)$, the scheme $\mathcal{S}=\left(\prod_{i=1}^{n} f_{i}\right)$ on a metric space $X$ is said to be $\alpha$-contractive if it satisfies the following conditions: 
(1) For each sequence $\left(t_{n}\right) \in\left\{\left(\prod_{i=0}^{n} f_{k+i}\right): k \in \mathbb{N}\right\}$,

$$
d\left(t_{n+1}(x), t_{n}(x)\right) \leq \alpha d\left(t_{n}(x), t_{n-1}(x)\right)
$$

for each $n \in \mathbb{N}$, where $t_{0}(x)=x$ for each $x \in X$.

(2) The set $\mathcal{F}=\left\{f_{n}: n \in \mathbb{N}\right\}$ is equicontinuous on $\operatorname{Fix}(\mathcal{F})$.

Example 2.21 If $f$ is an $\alpha$-contraction on a metric space, then the Picard iteration scheme $\left(f^{n}\right)$ is $\alpha$-contractive.

Theorem 2.22 Every $\alpha$-contractive scheme $\mathcal{S}$ on a metric space $X$ is virtually stable. Moreover, if $X$ is complete, then $\mathrm{C}(\mathcal{S})=X$.

Proof Let $\mathcal{S}=\left(\prod_{i=1}^{n} f_{i}\right)$ be an $\alpha$-contractive scheme. To show that $\mathcal{S}$ is virtually stable, let $p \in \operatorname{Fix}(\mathcal{S})=\operatorname{Fix}(\mathcal{F}), \varepsilon>0$, and $\varepsilon_{0}=\min \left\{\frac{\varepsilon(1-\alpha)}{4}, \frac{\varepsilon}{4}\right\}$. Since $\mathcal{F}$ is equicontinuous at $p \in \operatorname{Fix}(\mathcal{F})$, there exists $\delta>0$ such that

$$
f_{i}(B(p ; \delta)) \subseteq B\left(f_{i}(p) ; \varepsilon_{0}\right)
$$

for each $f_{i} \in \mathcal{F}$. WLOG, we may assume that $\delta \leq \varepsilon_{0}$.

Let $n \in \mathbb{N}, j \leq n, \mathcal{T}=\left(t_{n}\right)=\left(\prod_{i=0}^{n} f_{j+i}\right)$, and $y \in B(p ; \delta)$. Since $p \in \operatorname{Fix}(\mathcal{S}) \subseteq \operatorname{Fix}(\mathcal{T})$, we have $t_{1}(y)=f_{j}(y) \in B\left(f_{j}(p) ; \varepsilon_{0}\right)=B\left(p ; \varepsilon_{0}\right)$, and

$$
d\left(y, t_{1}(y)\right) \leq d(y, p)+d\left(p, t_{1}(y)\right)<\delta+\varepsilon_{0} \leq 2 \varepsilon_{0} \leq \frac{\varepsilon}{2} .
$$

Consequently,

$$
d\left(t_{1}(y), t_{n-j+1}(y)\right) \leq \sum_{i=1}^{n-j} d\left(t_{i}(y), t_{i-1}(y)\right) \leq \sum_{i=1}^{\infty} \alpha^{i-1} d\left(t_{1}(y), y\right) \leq 2 \varepsilon_{0}\left(\frac{1}{1-\alpha}\right) \leq \frac{\varepsilon}{2}
$$

So,

$$
\begin{aligned}
d\left(p, \prod_{i=j}^{n} f_{i}(y)\right) & \leq d\left(p, t_{1}(y)\right)+d\left(t_{1}(y), \prod_{i=j}^{n} f_{i}(y)\right) \\
& =d\left(p, t_{1}(y)\right)+d\left(t_{1}(y), t_{n-j+1}(y)\right)<\varepsilon .
\end{aligned}
$$

Hence, for each $p \in \operatorname{Fix}(\mathcal{S})$ and $\varepsilon>0$, there is $\delta>0$ such that

$$
\prod_{i=j}^{n} f_{i}(B(p ; \delta)) \subseteq B(p ; \varepsilon)
$$

for all $n \in \mathbb{N}$ and $j \leq n$. Therefore, $\mathcal{S}$ is virtually stable.

Notice that if a sequence $\left(x_{n}\right)$ satisfies $d\left(x_{n+1}, x_{n}\right) \leq \alpha d\left(x_{n}, x_{n-1}\right)$ for some $\alpha \in[0,1)$ and all $n \in \mathbb{N}$, then $\left(x_{n}\right)$ is a Cauchy sequence. This fact immediately implies that $\mathrm{C}(\mathcal{S})=X$ when $X$ is complete. 
Corollary 2.23 Let $X$ be a metric space and $f: X \rightarrow X$ continuous on Fix $(f)$. If the (Picard) scheme $\mathcal{S}=\left(f^{n}\right)$ satisfies

$$
d\left(f^{n+1}(x), f^{n}(x)\right) \leq \alpha d\left(f^{n}(x), f^{n-1}(x)\right)
$$

for some $\alpha \in[0,1)$ and for all $x \in X$, then $\mathcal{S}$ is $\alpha$-contractive and hence virtually stable.

Proof Observe that $\mathcal{F}=\{f\}$ is equicontinuous on $\operatorname{Fix}(\mathcal{F})$ by the continuity of $f$. Also, since $\left(\prod_{i=0}^{n} f_{k+i}\right)=\left(f^{n}\right)=\mathcal{S}$ for each $k \in \mathbb{N}$, then $\mathcal{S}$ is $\alpha$-contractive and hence virtually stable by the previous theorem.

\section{Main results}

We now present some new retraction and contractibility results for fixed point sets of certain set-valued mappings.

Theorem 3.1 Let $X$ be a closed subset of a Banach space E. If $F: X \rightarrow \operatorname{CCB}(X)$ is a lower semi-continuous quasi-nonexpansive mapping satisfying the end-point condition, then there is a virtually stable scheme $\mathcal{S}$ such that $\operatorname{Fix}(\mathcal{S})=\operatorname{Fix}(F)$, and hence $\operatorname{Fix}(F)$ is a retract of $\mathrm{C}(\mathcal{S})$.

Proof Note that $\mathrm{CC}(X) \subseteq \mathrm{CC}(E)$ because $X$ is closed in $E$. Since $F$ is lower semicontinuous, by Theorem 2.15, $F$ admits a continuous selection, say $f: X \rightarrow X$. By Lemma 2.8, $f$ is quasi-nonexpansive, and the scheme $\mathcal{S}=\left(f^{n}\right)$ is virtually stable by Example 2.18. Also, by $\operatorname{Proposition} 2.3, \operatorname{Fix}(\mathcal{S})=\operatorname{Fix}(f)=\operatorname{Fix}(F)$.

Corollary 3.2 Let $X$ be a closed subset of a Banach space. If $F: X \rightarrow \mathrm{CC}(X)$ is a *nonexpansive mapping satisfying the proximal condition, then there is a virtually stable scheme $\mathcal{S}$ such that $\operatorname{Fix}(\mathcal{S})=\operatorname{Fix}(F)$, and hence $\operatorname{Fix}(F)$ is a retract of $\mathrm{C}(\mathcal{S})$.

Proof From Lemma 2.4(1), (2) and Lemma 2.9, the mapping $P_{F}: X \rightarrow \operatorname{CCB}(X)$ is nonexpansive satisfying the end-point condition and $\operatorname{Fix}(F)=\operatorname{Fix}\left(P_{F}\right)$. Moreover, $P_{F}$ is lower semi-continuous by Lemma 2.5(2). Following Theorem 3.1, the sequence $\mathcal{S}=\left(f^{n}\right)$ is a virtually stable scheme with $\operatorname{Fix}(\mathcal{S})=\operatorname{Fix}(f)=\operatorname{Fix}(F)$, where $f$ is a continuous selection of $P_{F}$.

Theorem 3.3 Let $X$ be a compact metric space, and $F: X \rightarrow \mathrm{CB}(X)$ be a set-valued $\alpha$ contraction satisfying the Chebyshev condition. Then the sequence $\mathcal{S}=\left(s_{n}\right)$ of mappings on $X$, defined by

$$
s_{0}(x)=x \quad \text { and } \quad s_{n}(x)=P_{F}\left(s_{n-1}(x)\right)=\left(P_{F}\right)^{n}(x)
$$

for each $n \in \mathbb{N}$ and $x \in X$, is an $\alpha$-contractive scheme with $\operatorname{Fix}(\mathcal{S})=\operatorname{Fix}(F)$, and hence $\operatorname{Fix}(F)$ is a retract of $X$.

Proof Let $x \in X$ and $n \geq 1$. By Lemma 2.1(2) and (3),

$$
\begin{aligned}
d\left(s_{n-1}(x), s_{n}(x)\right) & =d\left(s_{n-1}(x), F \circ s_{n-1}(x)\right) \\
& \leq h\left(F \circ s_{n-2}(x), F \circ s_{n-1}(x)\right)
\end{aligned}
$$




$$
\begin{aligned}
& \leq H\left(F \circ s_{n-2}(x), F \circ s_{n-1}(x)\right) \\
& \leq \alpha d\left(s_{n-2}(x), s_{n-1}(x)\right) .
\end{aligned}
$$

It follows that $\left(s_{n}(x)\right)$ is a Cauchy sequence, and hence it converges to a point $p \in X$. Consider the following inequality:

$$
\begin{aligned}
d(p, F(p)) & \leq d\left(p, F \circ s_{n}(x)\right)+H\left(F \circ s_{n}(x), F(p)\right) \\
& \leq d\left(p, s_{n+1}(x)\right)+H\left(F \circ s_{n}(x), F(p)\right) .
\end{aligned}
$$

Since $\left(s_{n}(x)\right)$ converges to $p$ and $F$ is $H$-continuous, $\left(F \circ s_{n}(x)\right)$ converges to $F(p)$. Consequently, $d(p, F(p))=0$, and since $F(p)$ is closed, $p \in F(p)$. This implies $r(\mathrm{C}(\mathcal{S})) \subseteq \operatorname{Fix}(F)$. By Lemma 2.4(1),

$$
\operatorname{Fix}(F)=\operatorname{Fix}\left(P_{F}\right)=\operatorname{Fix}\left(\left\{\left(P_{F}\right)^{n}: n \in \mathbb{N}\right\}\right)=\operatorname{Fix}(\mathcal{S}) \subseteq r(C(\mathcal{S}))
$$

It follows that the sequence $\mathcal{S}$ is a scheme satisfying the condition in Corollary 2.23 with $\operatorname{Fix}(\mathcal{S})=\operatorname{Fix}(F)$. Therefore, the scheme $\mathcal{S}$ is $\alpha$-contractive with $\operatorname{Fix}(\mathcal{S})=\operatorname{Fix}(F)$, and hence Fix $(F)$ is a retract of $X$ by Lemma 2.22 and Theorem 2.19 .

Remark 3.4 The sequence $\left(\left(P_{F}\right)^{n}\right)$ in the proof of Theorem 3.3 is motivated by the iteration process defined in Theorem 5 [11]. Moreover, in [18], Yanagi studied the convergence of an iteration sequence for a sequence $\mathcal{F}=\left(F_{n}\right)$ of set-valued mappings from a complete metric space $X$ into $\mathrm{CB}(X)$ satisfying the relation

$$
\begin{aligned}
H\left(F_{i}(x), F_{j}(y)\right) \leq & a d(x, y)+b\left[d\left(x, F_{i}(x)\right)+d\left(y, F_{j}(y)\right)\right] \\
& +c\left[d\left(x, F_{j}(y)\right)+d\left(y, F_{i}(x)\right)\right]
\end{aligned}
$$

for all $x, y \in X$ and $i, j \in \mathbb{N}$, where $a, b, c \geq 0$ and $a+(a+3)(b+c)<1$. In this case, we observe that the sequence $\mathcal{S}=\left(\prod_{i=1}^{n} P_{F_{i}}\right)$ is an $\alpha$-contractive scheme for some $\alpha \in[0,1)$ if each $F_{i}$ satisfies the Chebyshev condition, and hence, by a similar argument in Theorem 3.3, the common fixed point set $\operatorname{Fix}(\mathcal{F})$ is a retract of $X$ when $X$ is compact.

Corollary 3.5 Let $X$ be a compact contractible metric space. If $F: X \rightarrow \mathrm{CB}(X)$ is an $\alpha$ contraction satisfying the Chebyshev condition, then $\operatorname{Fix}(F)$ is contractible.

Proof It follows directly from Theorem 3.3 and the contractibility of $X$.

The Chebyshev condition in Corollary 3.5 is necessary. For example, in Example 1 [11], the mapping $F$ is a contraction that does not satisfy the Chebyshev condition (consider $x=\frac{1}{3}$ ), and $\operatorname{Fix}(F)$ is clearly not contractible. Moreover, the following example shows that the fixed point set of an $\alpha$-contraction satisfying the Chebyshev condition may not be convex.

Example 3.6 Consider the subset $X=[-1,1] \times[0,1]$ of $\mathbb{R}^{2}$. Define $F: X \rightarrow \mathrm{CB}(X)$ by

$$
F(x, y)=[-1,1] \times\left\{\left|\frac{x}{2}\right|\right\} \quad \text { for each }(x, y) \in X .
$$


Then, for each $(x, y),\left(x_{1}, y_{1}\right),\left(x_{2}, y_{2}\right) \in X$,

$$
\begin{aligned}
H\left(F\left(x_{1}, y_{1}\right), F\left(x_{2}, y_{2}\right)\right) & =H\left([-1,1] \times\left\{\left|\frac{x_{1}}{2}\right|\right\},[-1,1] \times\left\{\left|\frac{x_{2}}{2}\right|\right\}\right) \\
& =|| \frac{x_{1}}{2}|-| \frac{x_{2}}{2}|| \\
& \leq \frac{1}{2}\left|x_{1}-x_{2}\right| \leq \frac{1}{2}\left\|\left(x_{1}, y_{1}\right)-\left(x_{2}, y_{2}\right)\right\|,
\end{aligned}
$$

and $P_{F}(x, y)=\left(x,\left|\frac{x}{2}\right|\right)$. Therefore, the mapping $F$ is a $\frac{1}{2}$-contraction satisfying the Chebyshev condition, and $\operatorname{Fix}(F)=\left\{(x, y): y=\left|\frac{x}{2}\right|\right\}$ which is not convex.

The following construction, motivated by the iteration process (1.3) in [12], indicates that there is a sequence of mappings that is not naturally a scheme, but still gives rise to a retraction result as well as a contractibility criterion for the fixed point set of a certain nonexpansive set-valued mapping.

Let $X$ be a closed convex subset of a Banach space, $F: X \rightarrow \operatorname{CCB}(X)$ be an $H$-continuous mapping, $\left(\alpha_{n}\right)_{n=0}^{\infty}$ be a sequence in $[a, b] \subseteq(0,1)$, and $\left(\gamma_{n}\right)_{n=0}^{\infty}$ be a sequence in $(0,+\infty)$ satisfying $\lim _{n \rightarrow \infty} \gamma_{n}=0$.

By Lemma 2.5(2) and Theorem 2.15, $F$ admits a continuous selection, say $g_{0}: X \rightarrow X$. We define mappings $s_{0}: X \rightarrow X$ and $s_{1}: X \rightarrow X$ by

$$
s_{0}(x)=x \quad \text { and } \quad s_{1}(x)=\left(1-\alpha_{0}\right) s_{0}(x)+\alpha_{0} g_{0}(x)
$$

for each $x \in X$, respectively.

To construct $s_{2}$, consider the mapping $\varphi: X \rightarrow(0, \infty)$ defined by

$$
\varphi(x)=H\left(F \circ s_{1}(x), F \circ s_{0}(x)\right)+\gamma_{0}
$$

for each $x \in X$. Let $x \in X$ and $\varepsilon>0$. Since $s_{1}$ and $s_{0}$ are continuous and $F$ is $H$-continuous, $F \circ s_{1}$ and $F \circ s_{0}$ are $H$-continuous. Then there is $\delta>0$ such that

$$
H\left(F \circ s_{1}(x), F \circ s_{1}(y)\right)<\frac{\varepsilon}{2} \quad \text { and } \quad H\left(F \circ s_{0}(y), F \circ s_{0}(x)\right)<\frac{\varepsilon}{2}
$$

for each $y \in B(x, \delta)$. Let $y \in B(x ; \delta)$, we may assume that $\varphi(x)-\varphi(y) \geq 0$, and hence

$$
\begin{aligned}
\varphi(x)-\varphi(y) \leq & H\left(F \circ s_{1}(x), F \circ s_{1}(y)\right)+H\left(F \circ s_{1}(y), F \circ s_{0}(y)\right) \\
& +H\left(F \circ s_{0}(y), F \circ s_{0}(x)\right)-H\left(F \circ s_{1}(y), F \circ s_{0}(y)\right) \\
= & H\left(F \circ s_{1}(x), F \circ s_{1}(y)\right)+H\left(F \circ s_{0}(y), F \circ s_{0}(x)\right)<\varepsilon .
\end{aligned}
$$

Therefore, $\varphi$ is continuous. Also, since $g_{0}(x) \in F(x)=F \circ s_{0}(x)$, then by Lemma 2.1(4), there exists $a \in F \circ s_{1}(x)$ such that $\left\|a-g_{0}(x)\right\|<\varphi(x)$. Thus,

$$
B\left(g_{0}(x) ; \varphi(x)\right) \cap F \circ s_{1}(x) \neq \varnothing .
$$


Following Lemma 2.16, there exists a continuous mapping $g_{1}: X \rightarrow X$ such that

$$
g_{1}(x) \in D\left(g_{0}(x) ; \varphi(x)\right) \cap F \circ s_{1}(x)
$$

for each $x \in X$. That is, $g_{1}$ is a continuous selection of $F \circ s_{1}$ satisfying

$$
\left\|g_{1}(x)-g_{0}(x)\right\| \leq H\left(F \circ s_{1}(x), F \circ s_{0}(x)\right)+\gamma_{0}
$$

for each $x \in X$. We now define $s_{2}: X \rightarrow X$ by

$$
s_{2}(x)=\left(1-\alpha_{1}\right) s_{1}(x)+\alpha_{1} g_{1}(x)
$$

for each $x \in X$.

Inductively, we obtain a sequence $\mathcal{S}=\left(s_{n}\right)$ of continuous mappings such that

$$
s_{n}(x)=\left(1-\alpha_{n-1}\right) s_{n-1}(x)+\alpha_{n-1} g_{n-1}(x)
$$

for each $x \in X$ and $n \geq 2$, where $g_{n-1}: X \rightarrow X$ is a continuous selection of $F \circ s_{n-1}$ satisfying

$$
\left\|g_{n-1}(x)-g_{n-2}(x)\right\| \leq H\left(F \circ s_{n-1}(x), F \circ s_{n-2}(x)\right)+\gamma_{n-1}
$$

for each $x \in X$.

Recall that $r: \mathrm{C}(\mathcal{S}) \rightarrow X$ is given by

$$
r(x)=\lim _{n \rightarrow \infty} s_{n}(x)
$$

for each $x \in \mathrm{C}(\mathcal{S})$.

Lemma 3.7 Suppose that $F: X \rightarrow \operatorname{CCB}(X)$ satisfies the end-point condition. Then $\operatorname{Fix}(F)=$ $\operatorname{Fix}(\mathcal{S})=r(\mathrm{C}(\mathcal{S}))$.

Proof It suffices to show that $\operatorname{Fix}(F) \subseteq \operatorname{Fix}(\mathcal{S})$ and $r(\mathrm{C}(\mathcal{S})) \subseteq \operatorname{Fix}(F)$.

Suppose $p \in \operatorname{Fix}(F)$. Since $g_{0}(p) \in F(p)=\{p\}$, we have

$$
s_{1}(p)=\left(1-\alpha_{0}\right) p+\alpha_{0} g_{0}(p)=p .
$$

Consequently, since $g_{1}(p) \in F \circ s_{1}(p)=\{p\}$,

$$
s_{2}(p)=\left(1-\alpha_{1}\right) s_{1}(p)+\alpha_{1} g_{1}(p)=p
$$

Inductively, for each $n \in \mathbb{N}, s_{n}(p)=p$. So, $p \in \bigcap_{n \in \mathbb{N}} \operatorname{Fix}\left(s_{n}\right)=\operatorname{Fix}(\mathcal{S})$.

Now, suppose $p \in r(\mathrm{C}(\mathcal{S}))$. Then $p=r(x)$ for some $x \in \mathrm{C}(\mathcal{S})$.

Since $s_{n+1}(x)-s_{n}(x)=\alpha_{n}\left(g_{n}(x)-s_{n}(x)\right),\left(\alpha_{n}\right)$ is a sequence in $[a, b] \subseteq(0,1)$ and $\left(s_{n}(x)\right)$ is a convergent sequence, we have

$$
\lim \sup _{n \rightarrow \infty}\left\|g_{n}(x)-s_{n}(x)\right\|=\lim \sup _{n \rightarrow \infty} \frac{\left\|s_{n+1}(x)-s_{n}(x)\right\|}{\alpha_{n}}=0 .
$$


Thus, $\lim _{n \rightarrow \infty}\left\|g_{n}(x)-s_{n}(x)\right\|=0$. For each $\varepsilon>0$, since $\left(s_{n}(x)\right)$ converges to $p$ and $F$ is $H$ continuous, there is $N \in \mathbb{N}$ such that $H\left(F \circ s_{n}(x), F(p)\right)<\varepsilon$ for all $n \geq N$. Consequently, $g_{n}(x) \in F \circ s_{n}(x) \subseteq \eta(F(p) ; \varepsilon)$ for all $n \geq N$. That is, $\lim _{n \rightarrow \infty} d\left(g_{n}(x), F(p)\right)=0$. Since, by Lemma 2.1(1),

$$
d(p, F(p)) \leq\left\|p-s_{n}(x)\right\|+\left\|s_{n}(x)-g_{n}(x)\right\|+d\left(g_{n}(x), F(p)\right),
$$

it follows that $d(p, F(p))=0$, and hence $p \in \operatorname{Fix}(F)$ since $F(p)$ is closed. Therefore, $r(\mathrm{C}(\mathcal{S})) \subseteq \operatorname{Fix}(F)$.

Lemma 3.8 Suppose that $F: X \rightarrow \operatorname{CCB}(X)$ is quasi-nonexpansive and satisfies the endpoint condition. Then, for each $x \in X, p \in \operatorname{Fix}(F)$ and $m \geq n$,

$$
\left\|s_{m}(x)-p\right\| \leq\left\|s_{n}(x)-p\right\|
$$

and $r$ is continuous.

Proof For each $x \in X, p \in \operatorname{Fix}(F)$ and $n \in \mathbb{N}$, we have

$$
\left\|g_{n}(x)-p\right\| \leq h\left(F \circ s_{n}(x),\{p\}\right) \leq H\left(F \circ s_{n}(x), F(p)\right) \leq\left\|s_{n}(x)-p\right\|,
$$

and hence

$$
\begin{aligned}
\left\|s_{n+1}(x)-p\right\| & =\left(1-\alpha_{n}\right)\left\|s_{n}(x)-p\right\|+\alpha_{n}\left\|g_{n}(x)-p\right\| \\
& \leq\left(1-\alpha_{n}\right)\left\|s_{n}(x)-p\right\|+\alpha_{n}\left\|s_{n}(x)-p\right\|=\left\|s_{n}(x)-p\right\| .
\end{aligned}
$$

Inductively, we have $\left\|s_{m}(x)-p\right\| \leq\left\|s_{n}(x)-p\right\|$ whenever $m \geq n$.

For the continuity of $r$, let $x \in \mathrm{C}(\mathcal{S})$ and $\varepsilon>0$. Then there exists $N \in \mathbb{N}$ such that $\left\|s_{N}(x)-r(x)\right\|<\frac{\varepsilon}{3}$, and by the continuity of $s_{N}$, there exists $\delta>0$ such that $\left\|s_{N}(x)-s_{N}(y)\right\|<$ $\frac{\varepsilon}{3}$ whenever $\|x-y\|<\delta$. Hence, for each $y \in \mathrm{C}(\mathcal{S})$ with $\|x-y\|<\delta$, there is $M \geq N$ such that $\left\|s_{M}(y)-r(y)\right\|<\frac{\varepsilon}{3}$. Observe that since $r(x) \in \operatorname{Fix}(F)$ and by the above property of $\left(s_{n}\right)$, we have

$$
\left\|r(x)-s_{M}(y)\right\| \leq\left\|r(x)-s_{N}(y)\right\|
$$

Then it follows that

$$
\begin{aligned}
\|r(x)-r(y)\| & \leq\left\|r(x)-s_{M}(y)\right\|+\left\|s_{M}(y)-r(y)\right\| \\
& \leq\left\|r(x)-s_{N}(y)\right\|+\left\|s_{M}(y)-r(y)\right\| \\
& \leq\left\|r(x)-s_{N}(x)\right\|+\left\|s_{N}(x)-s_{N}(y)\right\|+\left\|s_{M}(y)-r(y)\right\| \\
& <\varepsilon
\end{aligned}
$$

which proves the continuity of $r$.

By Lemma 3.7 and Lemma 3.8, we immediately obtain the following. 
Theorem 3.9 Let $X$ be a closed convex subset of a Banach space. If $F: X \rightarrow \operatorname{CCB}(X)$ is $H$-continuous, quasi-nonexpansive and satisfies the end-point condition, then $\operatorname{Fix}(F)$ is a retract of $\mathrm{C}(\mathcal{S})$, where the sequence $\mathcal{S}$ is defined as in (1).

Corollary 3.10 Let $X$ be a compact convex subset of a Banach space and $F: X \rightarrow \mathrm{CCB}(X)$ be a nonexpansive set-valued mapping satisfying the end-point condition. Then the fixed point set of $F$ is contractible.

Proof By Theorem 2.3 [12], we have $X=\mathrm{C}(\mathcal{S})$, and hence the result follows immediately from the previous theorem.

The example below shows that, under the assumption of the above corollary, the fixed point set may not be convex.

Example 3.11 Consider $X=[-1,1] \times[0,1] \subseteq \mathbb{R}^{2}$ with the maximum norm $\|(x, y)\|=$ $\max \{|x|,|y|\}$. Define $f: X \rightarrow X$ by

$$
f(x, y)=(x,|x|) \quad \text { for each }(x, y) \in \mathbb{R}^{2} .
$$

Then $f$ is a nonexpansive mapping which is also a nonexpansive set-valued mapping satisfying the end-point condition. Observe that $\operatorname{Fix}(f)=\{(x,|x|): x \in \mathbb{R}\}$ is not convex.

Motivated by the last construction, we are ready to extend the notion of fixed point iteration schemes to the notion of fixed point resolutions as follows.

Definition 3.12 For a (nonempty) Hausdorff space $X$, a sequence $\mathcal{S}=\left(s_{n}\right)$ of mappings of $X$ is said to be a fixed point resolution, or a resolution in short, if $r: C(\mathcal{S}) \rightarrow X$ given by

$$
r(x)=\lim _{n \rightarrow \infty} s_{n}(x)
$$

is continuous and $r(\mathrm{C}(\mathcal{S})) \subseteq \operatorname{Fix}(\mathcal{S})$.

Hence, the fixed point set of a resolution is always a retract of its convergence set, and when $X$ is a regular space, every virtually stable scheme on $X$ having a continuous subsequence is always a resolution. The last theorem below summarizes all retraction results in this section in terms of resolutions.

Theorem 3.13 Suppose that $X$ is a subset of a Banach space $E$ and $F: X \rightarrow Y \subseteq 2^{X}$ is a setvalued mapping. Then there exists a resolution $\mathcal{S}$ with $\operatorname{Fix}(\mathcal{S})=\operatorname{Fix}(F)$ if one the following conditions holds:

(1) $X$ is closed, $Y=\mathrm{CCB}(X)$ and $F$ is a quasi-nonexpansive and lower semi-continuous mapping satisfying the end-point condition.

(2) $X$ is closed, $Y=\mathrm{CC}(X)$ and $F$ is a *-nonexpansive mapping satisfying the proximal condition.

(3) $X$ is compact, $Y=\mathrm{CB}(X)$ and $F$ is a set-valued $\alpha$-contraction satisfying the Chebyshev condition.

(4) $X$ is closed and convex, $Y=\mathrm{CCB}(X)$ and $F$ is a nonexpansive mapping satisfying the end-point condition. 
Competing interests

The authors declare that they have no competing interests.

\section{Authors' contributions}

All authors contributed equally to the writing of this paper. All authors read and approved the final manuscript.

\section{Acknowledgements}

The authors would like to thank A Thamrongthanyalak for useful discussion during the manuscript preparation. The second author is (partially) supported by the Centre of Excellence in Mathematics, the Commission on Higher Education, Thailand.

Received: 23 December 2014 Accepted: 1 April 2015 Published online: 22 April 2015

\section{References}

1. Bruck, RE: Properties of fixed-point sets of nonexpansive mappings in Banach spaces. Trans. Am. Math. Soc. 179, 251-262 (1973)

2. Benavides, TD, Ramirez, PL: Structure of the fixed point set and common fixed points of asymptotically nonexpansive mappings. Proc. Am. Math. Soc. 129(12), 3549-3557 (2001)

3. Chaoha, P: Virtually nonexpansive maps and their convergence sets. J. Math. Anal. Appl. 326, 390-397 (2007)

4. Chaoha, P, Atiponrat, W: Virtually stable maps and their fixed point sets. J. Math. Anal. Appl. 359, 536-542 (2009)

5. Chaoha, P, Chanthorn, P: Fixed point sets through iteration schemes. J. Math. Anal. Appl. 386, 273-277 (2012)

6. Berinde, V: Iterative Approximation of Fixed Points, 2nd edn. Lecture Notes in Math. Springer, Berlin (2007)

7. Dhompongsa, S, Kaewkhao, A, Panyanak, B: On Kirk's strong convergence theorem for multivalued nonexpansive mappings on CAT(0) spaces. Nonlinear Anal. 75, 459-468 (2012)

8. Neammanee, K, Kaewkhao, A: On multi-valued weak contraction mappings. J. Math. Res. 3(2), 151-156 (2011)

9. Munkres, JR: Topology, 2nd edn. Prentice Hall, New York (2000)

10. Geletu, A: Introduction to Topological Spaces and Set-Valued Maps. Lecture Notes in Math. Institute of Mathematics, Department of Operations Research \& Stochestics, IImenau University of Technology (2006)

11. Nalder, SB: Multi-valued contraction mappings. Pac. J. Math. 30(2), 475-488 (1969)

12. Song, $\mathrm{Y}, \mathrm{Wang}, \mathrm{H}$ : Convergence of iterative algorithms for multivalued mappings in Banach spaces. Nonlinear Anal. 70, 1547-1556 (2009)

13. Li, J: The metric projection and its applications to solving variational inequalities in Banach spaces. Fixed Point Theory 5(2), 285-298 (2004)

14. Beg, I, Khan, AR, Hussain, N: Approximation of $*$-nonexpansive random multivalued operators on Banach spaces. J. Aust. Math. Soc. 76, 51-66 (2004)

15. Michael, E: Continuous selection. I. Ann. Math. 63(2), 361-382 (1956)

16. Thamrongthanyalak, A: Extensions and smooth approximations of definable functions in o-minimal structures. PhD thesis, University of California, Los Angeles (2013)

17. Assad, NA, Kirk, WA: Fixed point theorems for set-valued mappings of contractive type. Pac. J. Math. 43(3), 553-562 (1972)

18. Yanagi, K: A common fixed point theorem for a sequence of multivalued mappings. Publ. Res. Inst. Math. Sci. 15, 47-52 (1979)

\section{Submit your manuscript to a SpringerOpen ${ }^{\circ}$ journal and benefit from:}

- Convenient online submission

Rigorous peer review

- Immediate publication on acceptance

- Open access: articles freely available online

- High visibility within the field

- Retaining the copyright to your article 